\title{
Study of Vanadium Carbide Structures Based on Ve and Ev-Degree Topological Indices
}

\author{
Abdul Rauf ${ }^{(D)},{ }^{1}$ Saba Maqbool, ${ }^{1}$ Muhammad Naeem $\left(\mathbb{D},{ }^{1}\right.$ Adnan Aslam ${ }^{(D)},{ }^{2}$ Hamideh Aram, ${ }^{3}$ \\ and Kraidi Anoh Yannick $\mathbb{D}^{4}$ \\ ${ }^{1}$ Department of Mathematics, Air University Multan Campus, Multan, Pakistan \\ ${ }^{2}$ Department of Natural Sciences and Humanities, University of Engineering and Technology, Lahore, Pakistan (RCET), Pakistan \\ ${ }^{3}$ Department of Mathematics, Gareziaeddin Center, Khoy Branch, Islamic Azad University Khoy, Iran \\ ${ }^{4}$ UFR of Mathematics and Informatics, University Felix Houphouet Boigny of Cocody, Abidjan, Côte d'Ivoire
}

Correspondence should be addressed to Kraidi Anoh Yannick; kayanoh2000@yahoo.fr

Received 13 June 2021; Accepted 7 December 2021; Published 27 December 2021

Academic Editor: Muhammad Imran

Copyright ( $\odot 2021$ Abdul Rauf et al. This is an open access article distributed under the Creative Commons Attribution License, which permits unrestricted use, distribution, and reproduction in any medium, provided the original work is properly cited.

Vanadium is a biologically active product with significant industrial and biological applications. Vanadium is found in a variety of minerals and fossil fuels, the most common of which are sandstones, crude oil, and coal. Topological descriptors are numerical numbers assigned to the molecular structures and have the ability to predict certain of their physical/chemical properties. In this paper, we have studied topological descriptors of vanadium carbide structure based on ev and ve degrees. In particular, we have computed the closed forms of Zagreb, Randic, geometric-arithmetic, and atom-bond connectivity (ABC) indices of vanadium carbide structure based on ev and ve degrees. This kind of study may be useful for understanding the biological and chemical behavior of the structure.

\section{Introduction}

Vertex degree concept has devised many topological indices that are applicable in QSPR/QSAR studies. Topological indices are widely used in theoretical and mathematical chemistry as they are associated with the topology of a chemical structure along with its other identical properties such as boiling points, strain energy, and stability [1]. In chemical graph theory, a chemical graph is referred as a molecular structure with atoms as its vertices and chemical bonds as its edges. A topological index is a numerical parameter that creates a link between the physical and chemical properties of a molecule [2]. Many topological descriptors based on degree have been introduced. These topological indices have provided assistance in calculating different parametric calculations related to molecular structures to make them understandable and beneficial. A lot of topological descriptors have been defined and studied so far, but Zagreb indices [3], Weiner index [4], and Randic index [5] are the most studied among all of them. To read more about the chemical applicability of topological descriptors, see [5-12].

Researchers have attempted to study the varying behavior of transition metal carbides due to their complex structures. Such mineral metals are available in commercial places, and their salts are broadly utilized in our enterprises related to electrochemistry and material science. Among these, vanadium carbide complexes have shown crystal morphologies and stoichiometrics and display a great variety of superstructures. Very recently, many attempts have been done to purify high quality vanadium carbide by presenting different binary model system such as $V C, V_{2} C, V_{4} C_{3}, V_{6} C_{5}$, and $V_{8} C_{7}$. For more details, see [13-16].

Let $G$ be a simple connected graph with its edge set and vertex set denoted by $E$ and $V$, respectively. The neighbor set $N(v)$ of a vertex $v$ contains those vertices $v_{1}$ such that $v v_{1} \in E$. The degree of vertex $v$ is denoted by $d_{v}$ and is the cardinality of the set $N(v)$. Let $N[v]=\left\{v_{1} \in V: v v_{1} \in E\right\}$ 
$\cup\{v\}$ be the closed neighborhood of $v$. To read more about the basic concepts related to graph, see [17].

M. Chellali et al. [18] first introduced the concept of ev degree of an edge $e$ and ve degree of a vertex $v$. The ev degree of an edge $e$ is denoted by $d_{e v}(e)$ and is defined as the total number of vertices in the closed neighborhoods of the end vertices of an edge $e$. The ve degree of a vertex $v$ is denoted by $d_{v e}(v)$ and is the total number of edges that are adjacent with $v$ and the first neighbor of $v$. Ediz [3] first introduced the concept of ve degree and ev degree Zagreb and Randic indices. The mathematical formulas of these indices are presented in Table 1 . These newly defined indices were compared with Zagreb, Weiner, and Randic indices by modeling some of the physical/chemical properties of octane isomers. These indices have been observed to provide better correlation than the Randic, Weiner, and Zagreb indices for predicting some specific physical and chemical properties of octane isomers. Recently, a lot of work is done in the direction of computing newly defined ve degree and ev degreebased indices [19-23].

\section{Vanadium Carbide}

Vanadium carbide belongs to the family of group IV to VI transition metal carbides and shows homogeneity to metal nitrides, monocarbides, and carbonitrides. They possess unique associations between physio-chemical properties such as high melting points, high temperature resistivity, strength, and hardness which are associated with good electrical and thermal conductivity. These rare combinations of properties make such compounds very interesting for the researchers. These materials can be used as wear-resistant hard alloys and as hard coatings for protection purposes, due to their nanochemical properties [24, 25].

Vanadium carbide is the hardest inorganic metal-carbide with the formula VC. VC is an incredibly hard refractory ceramic with exceptional wear resistance, high modulus of elasticity ( $400 \mathrm{GPa})$, and good strength retention even at high temperatures [26-28]. VC coatings are used in corrosion prevention, cutting tool application, machining, drilling, and dyeing. Some industrial uses of VC are given in [27, 29-31]. We denote the crystallographic structure of vanadium carbide by $\operatorname{VC}[m, n]$. The molecular structure of vanadium carbide for $m=5=n$ is depicted in Figure 1. The structure of $\operatorname{VC}[m, n]$ has total number of $3 m n+m+n$ vertices and $6 m n-m-n$ edges. Let $V_{i}$ denote the vertex set containing the vertices of $V C[m, n]$ of degree $i$. Then, the vertex set $V(V C[m, n])$ can be partitioned into six sets with $\left|V_{1}\right|=m+n+2, \quad\left|V_{2}\right|=2 m+2 n-4, \quad\left|V_{3}\right|=4 m-2 n+2$, $\left|V_{4}\right|=1,\left|V_{5}\right|=m+n-2$, and $\left|V_{6}\right|=m n-m-n+1$. Let $E_{(i, j)}$ denote the edge set containing the edges of $\operatorname{VC}[m, n]$ with end vertices of degree $i$ and degree $j$. The edge set of $V C[m, n]$ can be partitioned based on the degree of end vertices as follows: $E_{(1,4)}$ with 1 edge, $E_{(1,5)}$ with 2 edges, $E_{(1,6)}$ with $m+n-1$ edges, $E_{(2,4)}$ with 2 edges, $E_{(2,5)}$ with $2 m+2 n-4$ edges, $E_{(2,6)}$ with $2 m+2 n-6$ edges, $E_{(3,4)}$ with 1 edge, $E_{(3,5)}$ with $3 m+3 n-8$ edges, and $E_{(3,6)}$ having $6 m n-9 m-9 n+13$ edges. In Theorem 1 , we compute the ev degree Randic and ev degree Zagreb index of $V C[m, n]$.

\section{Main Results}

Theorem 1. Let $m, n \geq 2$, then

$$
\begin{aligned}
\mathrm{M}^{e v}(V C[m, n])= & 486 m n-262 m-262 n+130 \\
\mathrm{R}^{e v}(V C[m, n])= & \frac{1}{\sqrt{7}} m n+\left(\frac{1}{\sqrt{7}}+\frac{2}{\sqrt{7}}+\frac{2}{\sqrt{8}}+\frac{3}{\sqrt{8}}-\frac{9}{\sqrt{9}}\right) m \\
& +\left(\frac{1}{\sqrt{7}}+\frac{2}{\sqrt{7}}+\frac{2}{\sqrt{8}}+\frac{3}{\sqrt{8}}-\frac{9}{\sqrt{9}}\right) n+\left(\frac{1}{\sqrt{5}}+\frac{4}{\sqrt{6}}-\frac{4}{\sqrt{7}}-\frac{6}{\sqrt{8}}-\frac{8}{\sqrt{8}}+\frac{13}{\sqrt{9}}\right) .
\end{aligned}
$$

Proof. To compute the ev degree Zagreb and ev degree Randic index of $V C[m, n]$, we need to compute the ev degree of the edges in each partition set $E_{(i, j)}$. This calculation is presented in Table 2. Now, using the information presented in Table 2 and the definition of ev degree Zagreb and ev degree Randic index, we get

$$
\begin{aligned}
\mathrm{M}^{e v}(V C[m, n])= & \sum_{e \in E(Z)} d_{e v}(e)^{2} \\
= & (5)^{2}\left|E_{(1,4)}\right|+(6)^{2}\left|E_{(1,5)}\right|+(7)^{2}\left|E_{(1,6)}\right|+(6)^{2}\left|E_{(2,4)}\right|+(7)^{2}\left|E_{(2,5)}\right| \\
& +(8)^{2}\left|E_{(2,6)}\right|+(7)^{2}\left|E_{(3,4)}\right|+(8)^{2}\left|E_{(3,5)}\right|+(9)^{2}\left|E_{(3,6)}\right| \\
= & (5)^{2}(1)+(6)^{2}(2)+(7)^{2}(m+n-l)+(6)^{2}(2)+(7)^{2}(2 m+2 n-4)
\end{aligned}
$$


TABLE 1: Mathematical formula of topological indices.

\begin{tabular}{lcc}
\hline Topological indices & Notation & Mathematical formula \\
\hline Ev degree Randic index & $\mathrm{R}^{e v}(G)$ & $\sum_{e \in E(G)} d_{e v}(e)^{-(1 / 2)}$ \\
First Zagreb $\beta$-index & $\mathrm{M}_{1}^{\beta v e}(G)$ & $\sum_{u v \in E(G)}\left(d_{v e}(u)+d_{v e}(v)\right)$ \\
Ev degree Zagreb index & $\mathrm{M}^{e v}(G)$ & $\sum_{e \in E(G)}(e)_{e v}\left(d^{2}\right.$ \\
Second Zagreb $\beta$-index & $\mathrm{M}_{2}^{\beta v e}(G)$ & $\sum_{u v \in E(G)}\left(d_{v e}(u) \times d_{v e}(v)\right)$ \\
Ve degree harmonic index & $H^{v e}(G)$ & $\sum_{u v \in E(G)}\left((2) /\left(d_{v e}(u)+d_{v e}(v)\right)\right)$ \\
Ve degree sum connectivity index & $\chi^{v e}(G)$ & $\sum_{u v \in E(G)}\left(d_{v e}(u)+d_{v e}(v)\right)^{-1 / 2}$ \\
Ve degree geometric arithmetic index & $G A^{v e}(G)$ & $\sum_{u v \in E(G)}\left(2 \sqrt{d_{v e}(u) \times d_{v e}(v)}\right) /\left(d_{v e}(u)+d_{v e}(v)\right)$ \\
Ve degree atom bond connectivity index & $A B C^{v e}(G)$ & $\sum_{u v \in E(G)} \sqrt{\left(d_{v e}(u)+d_{v e}(v)-2\right) /\left(d_{v e}(u) \times d_{v e}(v)\right)}$ \\
\hline
\end{tabular}

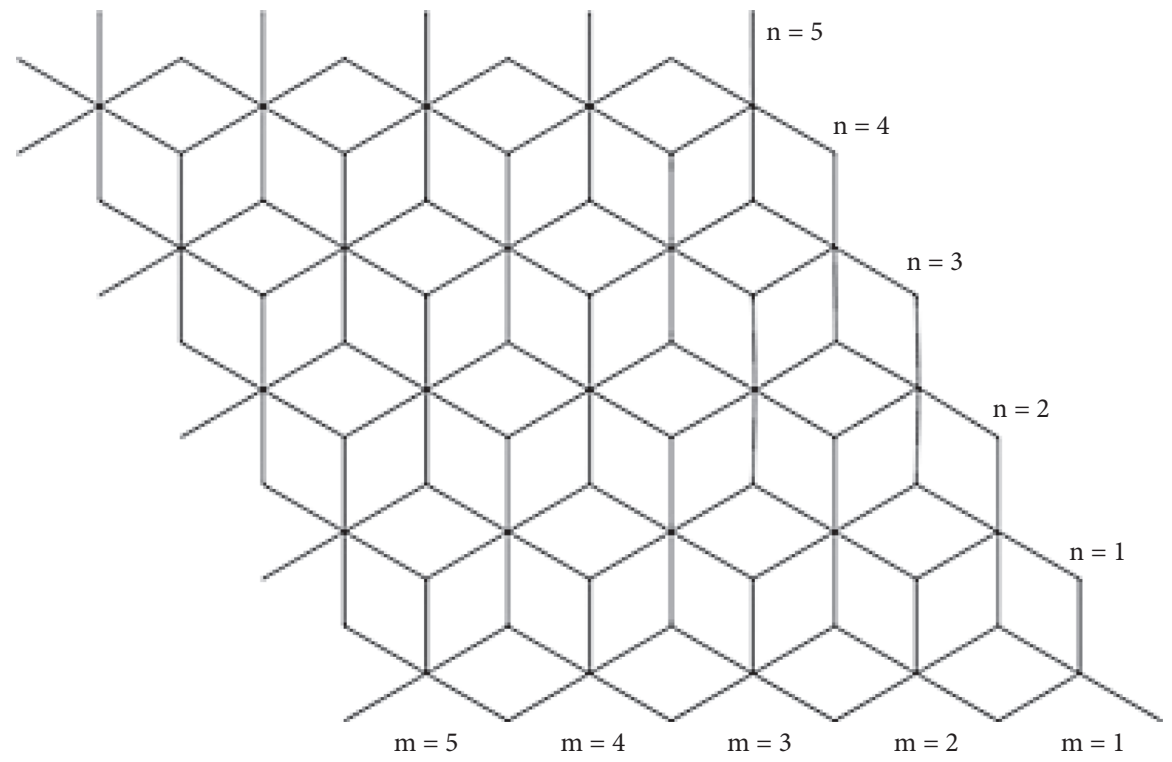

FIGURE 1: Molecular structure of vanadium carbide for $m=5$ and $n=5$.

TABLE 2: Ev-degrees of edges of vanadium carbide.

\begin{tabular}{lcc}
\hline$(d(u), d(v))$ & $d_{e v}(e)$ & Frequency \\
\hline$E_{(1,4)}$ & 5 & 1 \\
$E_{(1,5)}$ & 6 & 2 \\
$E_{(1,6)}$ & 7 & $m+n-1$ \\
$E_{(2,4)}$ & 6 & 2 \\
$E_{(2,5)}$ & 7 & $2 m+2 n-4$ \\
$E_{(2,6)}$ & 8 & $2 m+2 n-6$ \\
$E_{(3,4)}$ & 7 & $l$ \\
$E_{(3,5)}$ & 8 & $3 m+3 n-8$ \\
$E_{(3,6)}$ & 9 & $6 m n-9 m-9 n+13$ \\
\hline
\end{tabular}

$$
\begin{aligned}
& +(8)^{2}(2 m+2 n-6)+(9)^{2}(1)+(7)^{2}(3 m+3 n-8) \\
& +(8)^{2}(6 m n-9 m-9 n+13) \\
= & 486 m n-262 m-262 n+130 . \\
\mathrm{R}^{e v}(V C[m, n])= & \sum_{e \in E(Z)} d_{e v}(e)^{-1 / 2}, \\
= & (5)^{-1 / 2}\left|E_{(1,4)}\right|+(6)^{-1 / 2}\left|E_{(1,5)}\right|+(7)^{-1 / 2}\left|E_{(1,6)}\right|+(6)^{-1 / 2}\left|E_{(2,4)}\right|+(7)^{-1 / 2}\left|E_{(2,5)}\right| \\
& +(8)^{-1 / 2}\left|E_{(2,6)}\right|+(7)^{-1 / 2}\left|E_{(3,4)}\right|+(8)^{-1 / 2}\left|E_{(3,5)}\right|+(9)^{-1 / 2}\left|E_{(3,6)}\right|
\end{aligned}
$$




$$
\begin{aligned}
= & (5)^{-1 / 2}(1)+(6)^{-1 / 2}(2)+(7)^{-1 / 2}(m+n-l)+(6)^{-1 / 2}(2)+(7)^{-1 / 2}(2 m+2 n-4) \\
& +(8)^{-1 / 2}(2 m+2 n-6)+(9)^{-1 / 2}(1)+(7)^{-1 / 2}(3 m+3 n-8) \\
& +(8)^{-1 / 2}(6 m n-9 m-9 n+13) \\
= & \frac{1}{\sqrt{7}} m n+\left(\frac{1}{\sqrt{7}}+\frac{2}{\sqrt{7}}+\frac{2}{\sqrt{8}}+\frac{3}{\sqrt{8}}-\frac{9}{\sqrt{9}}\right) m+\left(\frac{1}{\sqrt{7}}+\frac{2}{\sqrt{7}}+\frac{2}{\sqrt{8}}+\frac{3}{\sqrt{8}}-\frac{9}{\sqrt{9}}\right) n \\
& +\left(\frac{1}{\sqrt{5}}+\frac{4}{\sqrt{6}}-\frac{4}{\sqrt{7}}-\frac{6}{\sqrt{8}}-\frac{8}{\sqrt{8}}+\frac{13}{\sqrt{9}}\right) .
\end{aligned}
$$

Theorem 2. Let $m, n \geq 3$, then

$$
\begin{aligned}
& \mathrm{M}_{1}^{\beta v e}(V C[m, n])=216 m n+76 m+20 n+54, \\
& \mathrm{M}_{2}^{\beta v e}(V C[m, n])=1944 m n-51 m-1887 n+1798 \text {, } \\
& \operatorname{ABC}^{v e}(V C[m, n])=\left(6 \sqrt{\frac{34}{324}}\right) m n+\left(\sqrt{\frac{18}{84}}+2 \sqrt{\frac{21}{130}}+2 \sqrt{\frac{24}{168}}+\sqrt{\frac{28}{121}}+2 \sqrt{\frac{27}{208}}+3 \sqrt{\frac{30}{252}}\right. \\
& \left.+\sqrt{\frac{32}{288}}+8 \sqrt{\frac{33}{306}}-15 \sqrt{\frac{34}{324}}\right) m+\left(\sqrt{\frac{18}{84}}+2 \sqrt{\frac{21}{130}}+2 \sqrt{\frac{24}{168}}+\sqrt{\frac{28}{121}}\right. \\
& \left.+2 \sqrt{\frac{27}{208}}+3 \sqrt{\frac{30}{252}}+\sqrt{\frac{32}{288}}+2 \sqrt{\frac{33}{306}}-15 \sqrt{\frac{34}{324}}\right) n+\left(\sqrt{\frac{10}{32}}+2 \sqrt{\frac{14}{55}}\right. \\
& +3 \sqrt{\frac{14}{60}}-4 \sqrt{\frac{18}{84}}+2 \sqrt{\frac{15}{72}}+2 \sqrt{\frac{19}{110}}+2 \sqrt{\frac{20}{121}}-10 \sqrt{\frac{21}{130}}+2 \sqrt{\frac{22}{117}} \\
& +2 \sqrt{\frac{23}{154}}+2 \sqrt{\frac{20}{120}}-10 \sqrt{\frac{24}{168}} \sqrt{\frac{22}{112}}-6 \sqrt{\frac{28}{121}}+2 \sqrt{\frac{25}{182}}-8 \sqrt{\frac{27}{208}}+2 \sqrt{\frac{25}{176}} \\
& \left.+2 \sqrt{\frac{26}{187}}+2 \sqrt{\frac{29}{238}}-14 \sqrt{\frac{30}{252}}-3 \sqrt{\frac{32}{288}}-10 \sqrt{\frac{33}{306}}+\sqrt{\frac{26}{180}}+37 \sqrt{\frac{34}{324}}\right) \text {, } \\
& \mathrm{GA}^{v e}(V C[m, n])=\frac{12 \sqrt{324}}{36} m n+\left(\frac{2 \sqrt{84}}{20}+\frac{4 \sqrt{130}}{23}+\frac{4 \sqrt{168}}{26}+\frac{2 \sqrt{121}}{30}+\frac{4 \sqrt{208}}{29}+\frac{6 \sqrt{252}}{32}\right. \\
& \left.+\frac{2 \sqrt{288}}{34}+\frac{16 \sqrt{306}}{35}-\frac{30 \sqrt{324}}{36}\right) m+\left(\frac{2 \sqrt{84}}{20}+\frac{4 \sqrt{130}}{23}+\frac{4 \sqrt{168}}{26}+\frac{2 \sqrt{121}}{30}\right. \\
& \left.+\frac{4 \sqrt{208}}{29}+\frac{6 \sqrt{252}}{32}+\frac{2 \sqrt{288}}{34}+\frac{4 \sqrt{306}}{35}-\frac{30 \sqrt{324}}{36}\right) n+\left(\frac{2 \sqrt{32}}{12}+\frac{4 \sqrt{55}}{16}\right. \\
& +\frac{6 \sqrt{60}}{16}-\frac{8 \sqrt{84}}{20}+\frac{4 \sqrt{72}}{17}+\frac{4 \sqrt{110}}{21}+\frac{4 \sqrt{121}}{22}-\frac{20 \sqrt{130}}{23}+\frac{4 \sqrt{117}}{22}+\frac{4 \sqrt{154}}{25} \\
& +\frac{4 \sqrt{120}}{22}-\frac{20 \sqrt{168}}{26}+\frac{2 \sqrt{112}}{22}-\frac{12 \sqrt{121}}{30}+\frac{4 \sqrt{182}}{27}-\frac{16 \sqrt{208}}{29}+\frac{4 \sqrt{176}}{27} \\
& \left.+\frac{4 \sqrt{187}}{28}+\frac{4 \sqrt{238}}{31}-\frac{28 \sqrt{252}}{32}-\frac{6 \sqrt{288}}{34}-\frac{20 \sqrt{306}}{35}+\frac{2 \sqrt{180}}{28}+\frac{74 \sqrt{324}}{36}\right) \text {, } \\
& \mathrm{H}^{v e}(V C[m, n])=\frac{1}{3} m n+\frac{10974905939}{29469607440} m+\frac{39532069}{247643760} n+\frac{801297510323}{950023374300} \text {, }
\end{aligned}
$$

$$
\begin{aligned}
\chi^{v e}(Z)= & m n+\left(\frac{1}{\sqrt{20}}+\frac{2}{\sqrt{23}}+\frac{2}{\sqrt{26}}+\frac{1}{\sqrt{30}}+\frac{2}{\sqrt{29}}+\frac{3}{\sqrt{32}}+\frac{1}{\sqrt{34}}+\frac{8}{\sqrt{35}}-\frac{15}{\sqrt{36}}\right) m+\left(\frac{1}{\sqrt{20}}\right. \\
& \left.+\frac{2}{\sqrt{23}}+\frac{2}{\sqrt{26}}+\frac{1}{\sqrt{30}}+\frac{2}{\sqrt{29}}+\frac{3}{\sqrt{32}}+\frac{1}{\sqrt{34}}+\frac{2}{\sqrt{35}}-\frac{15}{\sqrt{36}}\right) n+\left(\frac{1}{\sqrt{12}}+\frac{2}{\sqrt{16}}+\frac{3}{\sqrt{16}}\right. \\
& -\frac{4}{\sqrt{20}}+\frac{2}{\sqrt{17}}+\frac{2}{\sqrt{21}}+\frac{2}{\sqrt{22}}-\frac{10}{\sqrt{23}}+\frac{2}{\sqrt{22}}+\frac{2}{\sqrt{25}}-\frac{10}{\sqrt{26}}+\frac{1}{\sqrt{22}}-\frac{6}{\sqrt{30}}+\frac{2}{\sqrt{27}}
\end{aligned}
$$




$$
\begin{aligned}
& \left.-\frac{8}{\sqrt{29}}+\frac{2}{\sqrt{27}}+\frac{2}{\sqrt{28}}+\frac{2}{\sqrt{31}}-\frac{14}{\sqrt{32}}-\frac{3}{\sqrt{34}}-\frac{10}{\sqrt{35}}+\frac{1}{\sqrt{28}}+\frac{37}{\sqrt{36}}\right) \\
& \left.+\frac{8}{\sqrt{306}}-\frac{15}{\sqrt{324}}\right) m+\left(\frac{1}{\sqrt{84}}+\frac{2}{\sqrt{130}}+\frac{2}{\sqrt{168}}+\frac{1}{\sqrt{221}}+\frac{2}{\sqrt{208}}+\frac{3}{\sqrt{252}}\right. \\
& \left.+\frac{1}{\sqrt{288}}+\frac{2}{\sqrt{306}}-\frac{15}{\sqrt{324}}\right) n+\left(\frac{1}{\sqrt{32}}+\frac{2}{\sqrt{55}}+\frac{3}{\sqrt{60}}-\frac{1}{\sqrt{72}}+\frac{2}{\sqrt{110}}+\frac{2}{\sqrt{121}}\right. \\
& -\frac{4}{\sqrt{84}}-\frac{10}{\sqrt{130}}+\frac{2}{\sqrt{117}}+\frac{2}{\sqrt{154}}-\frac{10}{\sqrt{168}}+\frac{1}{\sqrt{112}}+\frac{6}{\sqrt{221}}+\frac{2}{\sqrt{182}}-\frac{8}{\sqrt{208}} \\
& \left.+\frac{2}{\sqrt{176}}+\frac{2}{\sqrt{187}}+\frac{2}{\sqrt{238}}-\frac{14}{\sqrt{252}}-\frac{3}{\sqrt{288}}-\frac{10}{\sqrt{300}}+\frac{1}{\sqrt{180}}+\frac{37}{\sqrt{324}}\right) .
\end{aligned}
$$

Proof. To compute the ve degree-based indices, we need to find the edge partition of $E(V C[m, n])$ based on the ve degree of end vertices of each edge. This partition is presented in Table 3. Now, using the values from Table 3 and the definition of ve degree indices, we get

$$
\begin{aligned}
& \mathrm{M}_{1}^{\beta v e}(V C[m, n])=\sum_{u v \in E(V C[m, n])}\left(d_{v e}(u)+d_{v e}(v)\right) \\
& =(12)\left|E_{1}\right|+(16)\left|E_{2}\right|+(16)\left|E_{3}\right|+(20)\left|E_{4}\right|+(17)\left|E_{5}\right|+(21)\left|E_{6}\right| \\
& +(22)\left|E_{7}\right|+(23)\left|E_{8}\right|+(22)\left|E_{9}\right|+(25)\left|E_{10}\right|+(22)\left|E_{11}\right|+(26)\left|E_{12}\right|+(22)\left|E_{13}\right| \\
& +(30)\left|E_{14}\right|+(27)\left|E_{15}\right|+(29)\left|E_{16}\right|+(27)\left|E_{17}\right|+(28)\left|E_{18}\right| \\
& +(31)\left|E_{19}\right|+(32)\left|E_{20}\right|+(34)\left|E_{21}\right|+(35)\left|E_{22}\right| \\
& +(28)\left|E_{23}\right|+(36)\left|E_{24}\right| \\
& =(12)(1)+(16)(2)+(16)(3)+(20)(m+n-4)+(17)(2)+(21)(2)+(22)(2) \\
& +(23)(2 m+2 n-10)+(22)(2)+(25)(2)+(22)(2)+(26)(2 m+2 n-10) \\
& +(22)(1)+(30)(m+n-6)+(27)(2)+(29)(2 m+2 n-8)+(27)(2)+(28)(2) \\
& +(31)(2)+(32)(3 m+3 n-14)+(34)(m+n-3)+(35)(8 m+2 n-10) \\
& +(28)(1)+(36)(6 m n-15 m-15 n+37) \\
& =216 m n+76 m+20 n+54, \\
& \mathrm{M}_{2}^{\beta v e}(V C[m, n])=\sum_{u v \in E(V C[m, n])}\left(d_{v e}(u) \times d_{v e}(v)\right) \\
& =(32)\left|E_{1}\right|+(55)\left|E_{2}\right|+(60)\left|E_{3}\right|+(84)\left|E_{4}\right|+(72)\left|E_{5}\right|+(110)\left|E_{6}\right| \\
& +(168)\left|E_{12}\right|+(112)\left|E_{13}\right|+(121)\left|E_{7}\right|+(130)\left|E_{8}\right|+(117)\left|E_{9}\right| \\
& +(154)\left|E_{10}\right|+(120)\left|E_{11}\right|+(121)\left|E_{14}\right|+(182)\left|E_{15}\right|+(208)\left|E_{16}\right| \\
& +(176)\left|E_{17}\right|+(187)\left|E_{18}\right|+(238)\left|E_{19}\right|+(252)\left|E_{20}\right|+(288)\left|E_{21}\right|
\end{aligned}
$$


Table 3: Edge Partition of vanadium carbide.

\begin{tabular}{|c|c|c|}
\hline Edge & $\left(d_{v e}(u), d_{v e}(v)\right)$ & Frequency \\
\hline$E_{1}$ & $(4,8)$ & 1 \\
\hline$E_{2}$ & $(5,11)$ & 2 \\
\hline$E_{3}$ & $(6,10)$ & 3 \\
\hline$E_{4}$ & $(6,14)$ & $m+n-4$ \\
\hline$E_{5}$ & $(9,8)$ & 2 \\
\hline$E_{6}$ & $(10,11)$ & 2 \\
\hline$E_{7}$ & $(11,11)$ & 2 \\
\hline$E_{8}$ & $(10,13)$ & $2 m+2 n-10$ \\
\hline$E_{9}^{\circ}$ & $(9,13)$ & 2 \\
\hline$E_{10}$ & $(11,14)$ & 2 \\
\hline$E_{11}$ & $(12,10)$ & 2 \\
\hline$E_{12}$ & $(12,14)$ & $2 m+2 n-10$ \\
\hline$E_{13}$ & $(8,14)$ & 1 \\
\hline$E_{14}$ & $(17,13)$ & $m+n-6$ \\
\hline$E_{15}$ & $(14,13)$ & 2 \\
\hline$E_{16}$ & $(16,13)$ & $2 m+2 n-8$ \\
\hline$E_{17}$ & $(16,11)$ & 2 \\
\hline$E_{18}$ & $(17,11)$ & 2 \\
\hline$E_{19}$ & $(17,14)$ & 2 \\
\hline$E_{20}$ & $(18,14)$ & $3 m+3 n-14$ \\
\hline$E_{21}$ & $(16,18)$ & $m+n-3$ \\
\hline$E_{22}$ & $(17,18)$ & $2 m+2 n-10$ \\
\hline$E_{23}$ & $(18,10)$ & 1 \\
\hline$E_{24}$ & $(18,18)$ & $6 m n-15 m-15 n+37$ \\
\hline
\end{tabular}

$$
\begin{aligned}
& +(306)\left|E_{22}\right|+(180)\left|E_{23}\right|+(324)\left|E_{24}\right| \\
= & (32)(1)+(55)(2)+(60)(3)+(84)(m+n-4)+(72)(2)+(110)(2)+(121)(2) \\
& +(130)(2 m+2 n-10)+(117)(2)+(154)(2)+(120)(2)+(168)(2 m+2 n-10) \\
& +(112)(1)+(121)(m+n-6)+(182)(2)+(208)(2 m+2 n-8)+(176)(2) \\
& +(187)(2)+(238)(2)+(252)(3 m+3 n-14)+(288)(m+n-3) \\
& +(306)(8 m+2 n-10)+(180)(1)+(324)(6 m n-15 m-15 n+37) \\
= & 1944 m n-51 m-1887 n+1798 .
\end{aligned}
$$

$$
\begin{aligned}
\operatorname{ABC}^{v e}(V C[m, n])= & \sum_{u v \in E(V C[m, n])} \sqrt{\frac{d_{v e}(u)+d_{v e}(v)-2}{\left(d_{v e}(u) \times d_{v e}(v)\right)}} \\
= & \left(\sqrt{\frac{10}{32}}\right)\left|E_{1}\right|+\left(\sqrt{\frac{14}{55}}\right)\left|E_{2}\right|+\left(\sqrt{\frac{14}{60}}\right)\left|E_{3}\right|+\left(\sqrt{\frac{18}{84}}\right)\left|E_{4}\right|+\left(\sqrt{\frac{21}{130}}\right)\left|E_{8}\right| \\
& +\left(\sqrt{\frac{15}{72}}\right)\left|E_{5}\right|+\left(\sqrt{\frac{19}{110}}\right)\left|E_{6}\right|+\left(\sqrt{\frac{20}{121}}\right)\left|E_{7}\right|+\left(\sqrt{\frac{22}{117}}\right)\left|E_{9}\right| \\
& +\left(\sqrt{\frac{23}{154}}\right)\left|E_{10}\right|+\left(\sqrt{\frac{20}{120}}\right)\left|E_{11}\right|+\left(\sqrt{\frac{24}{168}}\right)\left|E_{12}\right|+\left(\sqrt{\frac{22}{112}}\right)\left|E_{13}\right| \\
& +\left(\sqrt{\frac{28}{121}}\right)\left|E_{14}\right|+\left(\sqrt{\frac{25}{182}}\right)\left|E_{15}\right|+\left(\sqrt{\frac{27}{208}}\right)\left|E_{16}\right|+\left(\sqrt{\frac{25}{176}}\right)\left|E_{17}\right| \\
& +\left(\sqrt{\frac{26}{187}}\right)\left|E_{18}\right|+\left(\sqrt{\frac{29}{238}}\right)\left|E_{19}\right|+\left(\sqrt{\frac{30}{252}}\right)\left|E_{20}\right|+\left(\sqrt{\frac{32}{288}}\right)\left|E_{21}\right| \\
& +\left(\sqrt{\frac{33}{306}}\right)\left|E_{22}\right|+\left(\sqrt{\frac{26}{180}}\right)\left|E_{23}\right|+\left(\sqrt{\frac{34}{324}}\right)\left|E_{24}\right| \\
= & \left(6 \sqrt{\frac{34}{324}}\right) m n+\left(\sqrt{\frac{18}{84}+2} \sqrt{\frac{21}{130}}+2 \sqrt{\frac{24}{168}}+\sqrt{\frac{28}{121}}+2 \sqrt{\frac{27}{208}}+3 \sqrt{\frac{30}{252}}\right.
\end{aligned}
$$




$$
\begin{aligned}
& \left.+\sqrt{\frac{32}{288}}+8 \sqrt{\frac{33}{306}}-15 \sqrt{\frac{34}{324}}\right) m+\left(\sqrt{\frac{18}{84}}+2 \sqrt{\frac{21}{130}}+2 \sqrt{\frac{24}{168}}+\sqrt{\frac{28}{121}}\right. \\
& \left.+2 \sqrt{\frac{27}{208}}+3 \sqrt{\frac{30}{252}}+\sqrt{\frac{32}{288}}+2 \sqrt{\frac{33}{306}}-15 \sqrt{\frac{34}{324}}\right) n+\left(\sqrt{\frac{10}{32}}+2 \sqrt{\frac{14}{55}}\right. \\
& +3 \sqrt{\frac{14}{60}}-4 \sqrt{\frac{18}{84}}+2 \sqrt{\frac{15}{72}}+2 \sqrt{\frac{19}{110}}+2 \sqrt{\frac{20}{121}}-10 \sqrt{\frac{21}{130}}+2 \sqrt{\frac{22}{117}} \\
& +2 \sqrt{\frac{23}{154}}+2 \sqrt{\frac{20}{120}}-10 \sqrt{\frac{24}{168}}+\sqrt{\frac{22}{112}}-6 \sqrt{\frac{28}{121}}+2 \sqrt{\frac{25}{182}}-8 \sqrt{\frac{27}{208}} \\
& +2 \sqrt{\frac{25}{176}}+2 \sqrt{\frac{26}{187}}+2 \sqrt{\frac{29}{238}}-14 \sqrt{\frac{30}{252}}-3 \sqrt{\frac{32}{288}}-10 \sqrt{\frac{33}{306}}+\sqrt{\frac{26}{180}} \\
& \left.+37 \sqrt{\frac{34}{324}}\right),
\end{aligned}
$$

$$
\begin{aligned}
& \mathrm{GA}^{v e}(V C[m, n])= \sum_{u v \in E(V C[m, n])} \frac{2 \sqrt{d_{v e}(u) \times d_{v e}(v)}}{\left(d_{v e}(u)+d_{v e}(v)\right)} \\
&=\left(\frac{2 \sqrt{32}}{12}\right)\left|E_{1}\right|+\left(\frac{2 \sqrt{55}}{16}\right)\left|E_{2}\right|+\left(\frac{2 \sqrt{60}}{16}\right)\left|E_{3}\right|+\left(\frac{2 \sqrt{84}}{20}\right)\left|E_{4}\right|+\left(\frac{2 \sqrt{72}}{17}\right)\left|E_{5}\right| \\
&+\left(\frac{2 \sqrt{110}}{21}\right)\left|E_{6}\right|+\left(\frac{2 \sqrt{121}}{22}\right)\left|E_{7}\right|+\left(\frac{2 \sqrt{130}}{23}\right)\left|E_{8}\right|+\left(\frac{2 \sqrt{117}}{22}\right)\left|E_{9}\right| \\
&+\left(\frac{2 \sqrt{154}}{25}\right)\left|E_{10}\right|+\left(\frac{2 \sqrt{120}}{22}\right)\left|E_{11}\right|+\left(\frac{2 \sqrt{168}}{26}\right)\left|E_{12}\right|+\left(\frac{2 \sqrt{112}}{22}\right)\left|E_{13}\right| \\
&+\left(\frac{2 \sqrt{121}}{30}\right)\left|E_{14}\right|+\left(\frac{2 \sqrt{182}}{27}\right)\left|E_{15}\right|+\left(\frac{2 \sqrt{208}}{29}\right)\left|E_{16}\right|+\left(\frac{2 \sqrt{176}}{27}\right)\left|E_{17}\right| \\
&+\left(\frac{2 \sqrt{187}}{28}\right)\left|E_{18}\right|+\left(\frac{2 \sqrt{238}}{31}\right)\left|E_{19}\right|+\left(\frac{2 \sqrt{252}}{32}\right)\left|E_{20}\right|+\left(\frac{2 \sqrt{288}}{34}\right)\left|E_{21}\right| \\
&+\left(\frac{2 \sqrt{306}}{35}\right)\left|E_{22}\right|+\left(\frac{2 \sqrt{180}}{28}\right)\left|E_{23}\right|+\left(\frac{2 \sqrt{324}}{36}\right)\left|E_{24}\right| \\
&= \frac{12 \sqrt{324}}{36} m n+\left(\frac{2 \sqrt{84}}{20}+\frac{4 \sqrt{130}}{23}+\frac{4 \sqrt{168}}{26}+\frac{2 \sqrt{121}}{30}+\frac{4 \sqrt{208}}{29}+\frac{6 \sqrt{252}}{32}\right. \\
&\left.+\frac{2 \sqrt{288}}{34}+\frac{16 \sqrt{306}}{35}-\frac{30 \sqrt{324}}{36}\right) m+\left(\frac{2 \sqrt{84}}{20}+\frac{4 \sqrt{130}}{23}+\frac{4 \sqrt{168}}{26}+\frac{2 \sqrt{121}}{30}\right. \\
&\left.+\frac{4 \sqrt{208}}{29}+\frac{6 \sqrt{252}}{32}+\frac{2 \sqrt{288}}{34}+\frac{4 \sqrt{306}}{35}-\frac{30 \sqrt{324}}{36}\right) n+\left(\frac{2 \sqrt{32}}{12}+\frac{4 \sqrt{55}}{16}\right. \\
&+\frac{6 \sqrt{60}}{16}-\frac{8 \sqrt{84}}{20}+\frac{4 \sqrt{72}}{17}+\frac{4 \sqrt{110}}{21}+\frac{4 \sqrt{121}}{22}-\frac{20 \sqrt{130}}{23}+\frac{4 \sqrt{117}}{22}+\frac{4 \sqrt{154}}{25} \\
&+\frac{4 \sqrt{120}}{22}-\frac{20 \sqrt{168}}{26}+\frac{2 \sqrt{112}}{22}-\frac{12 \sqrt{121}}{30}+\frac{4 \sqrt{182}}{27}-\frac{16 \sqrt{208}}{29}+\frac{4 \sqrt{176}}{27} \\
&\left.+\frac{4 \sqrt{187}}{28}+\frac{4 \sqrt{238}}{31}-\frac{28 \sqrt{252}}{32}-\frac{6 \sqrt{288}}{34}-\frac{20 \sqrt{306}}{35}+\frac{2 \sqrt{180}}{28}+\frac{74 \sqrt{324}}{36}\right) . \\
&
\end{aligned}
$$

$$
\begin{aligned}
\mathrm{H}^{v e}(V C[m, n])= & \sum_{u v \in E(V C[m, n])} \frac{2}{d_{v e}(u)+d_{v e}(v)} \\
= & \left(\frac{2}{12}\right)\left|E_{1}\right|+\left(\frac{2}{16}\right)\left|E_{2}\right|+\left(\frac{2}{16}\right)\left|E_{3}\right|+\left(\frac{2}{20}\right)\left|E_{4}\right|+\left(\frac{2}{17}\right)\left|E_{5}\right|+\left(\frac{2}{21}\right)\left|E_{6}\right| \\
& +\left(\frac{2}{22}\right)\left|E_{7}\right|+\left(\frac{2}{23}\right)\left|E_{8}\right|+\left(\frac{2}{22}\right)\left|E_{9}\right|+\left(\frac{2}{25}\right)\left|E_{10}\right|+\left(\frac{2}{22}\right)\left|E_{11}\right|+\left(\frac{2}{26}\right)\left|E_{12}\right| \\
& +\left(\frac{2}{22}\right)\left|E_{13}\right|+\left(\frac{2}{30}\right)\left|E_{14}\right|+\left(\frac{2}{27}\right)\left|E_{15}\right|+\left(\frac{2}{29}\right)\left|E_{16}\right|+\left(\frac{2}{27}\right)\left|E_{17}\right|
\end{aligned}
$$




$$
\begin{aligned}
& +\left(\frac{2}{28}\right)\left|E_{18}\right|+\left(\frac{2}{31}\right)\left|E_{19}\right|+\left(\frac{2}{32}\right)\left|E_{20}\right|+\left(\frac{2}{34}\right)\left|E_{21}\right|+\left(\frac{2}{35}\right)\left|E_{22}\right| \\
& +\left(\frac{2}{28}\right)\left|E_{23}\right|+\left(\frac{2}{36}\right)\left|E_{24}\right| \\
& =\frac{1}{3} m n+\frac{10974905939}{29469607440} m+\frac{39532069}{247643760} n+\frac{801297510323}{950023374300} \text {, } \\
& \chi^{v e}(V C[m, n])=\sum_{u v \in E(V C[m, n])}\left(d_{v e}(u)+d_{v e}(v)\right)^{-1 / 2} \\
& =(12)^{-1 / 2}\left|E_{1}\right|+(16)^{-1 / 2}\left|E_{2}\right|+(16)^{-1 / 2}\left|E_{3}\right|+(20)^{-1 / 2}\left|E_{4}\right|+(17)^{-1 / 2}\left|E_{5}\right| \\
& +(21)^{-1 / 2}\left|E_{6}\right|+(22)^{-1 / 2}\left|E_{7}\right|+(23)^{-1 / 2}\left|E_{8}\right|+(22)^{-1 / 2}\left|E_{9}\right|+(25)^{-1 / 2}\left|E_{10}\right| \\
& +(22)^{-1 / 2}\left|E_{11}\right|+(26)^{-1 / 2}\left|E_{12}\right|+(22)^{-1 / 2}\left|E_{13}\right|+(30)^{-1 / 2}\left|E_{14}\right| \\
& +(27)^{-1 / 2}\left|E_{15}\right|+(29)^{-1 / 2}\left|E_{16}\right|+(27)^{-1 / 2}\left|E_{17}\right|+(28)^{-1 / 2}\left|E_{18}\right| \\
& +(31)^{-1 / 2}\left|E_{19}\right|+(32)^{-1 / 2}\left|E_{20}\right|+(34)^{-1 / 2}\left|E_{21}\right|+(35)^{-1 / 2}\left|E_{22}\right| \\
& +(28)^{-1 / 2}\left|E_{23}\right|+(36)^{-1 / 2}\left|E_{24}\right| \\
& =m n+\left(\frac{1}{\sqrt{20}}+\frac{2}{\sqrt{23}}+\frac{2}{\sqrt{26}}+\frac{1}{\sqrt{30}}+\frac{2}{\sqrt{29}}+\frac{3}{\sqrt{32}}+\frac{1}{\sqrt{34}}+\frac{8}{\sqrt{35}}-\frac{15}{\sqrt{36}}\right) m \\
& +\left(\frac{1}{\sqrt{20}}+\frac{2}{\sqrt{23}}+\frac{2}{\sqrt{26}}+\frac{1}{\sqrt{30}}+\frac{2}{\sqrt{29}}+\frac{3}{\sqrt{32}}+\frac{1}{\sqrt{34}}+\frac{2}{\sqrt{35}}-\frac{15}{\sqrt{36}}\right) n \\
& +\left(\frac{1}{\sqrt{12}}+\frac{2}{\sqrt{16}}+\frac{3}{\sqrt{16}}-\frac{4}{\sqrt{20}}+\frac{2}{\sqrt{17}}+\frac{2}{\sqrt{21}}+\frac{2}{\sqrt{22}}-\frac{10}{\sqrt{23}}+\frac{2}{\sqrt{22}}+\frac{2}{\sqrt{25}}\right. \\
& -\frac{10}{\sqrt{26}}+\frac{1}{\sqrt{22}}-\frac{6}{\sqrt{30}}+\frac{2}{\sqrt{27}}-\frac{8}{\sqrt{29}}+\frac{2}{\sqrt{27}}+\frac{2}{\sqrt{28}}+\frac{2}{\sqrt{31}}-\frac{14}{\sqrt{32}}-\frac{3}{\sqrt{34}} \\
& \left.-\frac{10}{\sqrt{35}}+\frac{1}{\sqrt{28}}+\frac{37}{\sqrt{36}}\right) \text {. } \\
& \mathrm{R}^{v e}(V C[m, n])=\sum_{u v \in E(V C[m, n])}\left(d_{v e}(u) \times d_{v e}(v)\right)^{-1 / 2} \\
& =(32)^{-1 / 2}\left|E_{1}\right|+(55)^{-1 / 2}\left|E_{2}\right|+(60)^{-1 / 2}\left|E_{3}\right|+(84)^{-1 / 2}\left|E_{4}\right|+(72)^{-1 / 2}\left|E_{5}\right| \\
& +(110)^{-1 / 2}\left|E_{6}\right|+(121)^{-1 / 2}\left|E_{7}\right|+(130)^{-1 / 2}\left|E_{8}\right|+(117)^{-1 / 2}\left|E_{9}\right| \\
& +(154)^{-1 / 2}\left|E_{10}\right|+(120)^{-1 / 2}\left|E_{11}\right|+(168)^{-1 / 2}\left|E_{12}\right|+(112)^{-1 / 2}\left|E_{13}\right| \\
& +(121)^{-1 / 2}\left|E_{14}\right|+(182)^{-1 / 2}\left|E_{15}\right|+(208)^{-1 / 2}\left|E_{16}\right|+(176)^{-1 / 2}\left|E_{17}\right| \\
& +(187)^{-1 / 2}\left|E_{18}\right|+(238)^{-1 / 2}\left|E_{19}\right|+(252)^{-1 / 2}\left|E_{20}\right|+(288)^{-1 / 2}\left|E_{21}\right| \\
& +(306)^{-1 / 2}\left|E_{22}\right|+(180)^{-1 / 2}\left|E_{23}\right|+(324)^{-1 / 2}\left|E_{24}\right| \\
& =\frac{6}{\sqrt{324}} m n+\left(\frac{1}{\sqrt{84}}+\frac{2}{\sqrt{130}}+\frac{2}{\sqrt{168}}+\frac{1}{\sqrt{221}}+\frac{2}{\sqrt{208}}+\frac{3}{\sqrt{252}}+\frac{1}{\sqrt{288}}\right. \\
& \left.+\frac{8}{\sqrt{306}}-\frac{15}{\sqrt{324}}\right) m+\left(\frac{1}{\sqrt{84}}+\frac{2}{\sqrt{130}}+\frac{2}{\sqrt{168}}+\frac{1}{\sqrt{221}}+\frac{2}{\sqrt{208}}+\frac{3}{\sqrt{252}}\right. \\
& \left.+\frac{1}{\sqrt{288}}+\frac{2}{\sqrt{306}}-\frac{15}{\sqrt{324}}\right) n+\left(\frac{1}{\sqrt{32}}+\frac{2}{\sqrt{55}}+\frac{3}{\sqrt{60}}-\frac{4}{\sqrt{72}}+\frac{2}{\sqrt{110}}\right. \\
& +\frac{2}{\sqrt{121}}-\frac{4}{\sqrt{84}}-\frac{10}{\sqrt{130}}+\frac{2}{\sqrt{117}}+\frac{2}{\sqrt{154}}-\frac{10}{\sqrt{168}}+\frac{1}{\sqrt{112}}+\frac{6}{\sqrt{221}} \\
& +\frac{2}{\sqrt{182}}-\frac{8}{\sqrt{208}}+\frac{2}{\sqrt{176}}+\frac{2}{\sqrt{187}}+\frac{2}{\sqrt{238}}-\frac{14}{\sqrt{252}}-\frac{3}{\sqrt{288}}-\frac{10}{\sqrt{300}} \\
& \left.+\frac{1}{\sqrt{180}}+\frac{37}{\sqrt{324}}\right) \text {. }
\end{aligned}
$$


TABLE 4: Numerical results of indices for vanadium carbide.

\begin{tabular}{|c|c|c|c|c|c|}
\hline$[m, n]$ & $\mathrm{M}^{e v}(H)$ & $\mathrm{R}^{e v}(H)$ & $\mathrm{M}_{1}^{\alpha v e}(H)$ & $\mathrm{M}_{1}^{\beta v e}(H)$ & $\mathrm{M}_{2}^{\beta v e}(H)$ \\
\hline$[1,1]$ & 92 & 0.1332 & 1324 & 2110 & 1804 \\
\hline$[2,2]$ & 1026 & 1.0704 & 2732 & 2854 & 5698 \\
\hline$[3,3]$ & 2932 & 2.7636 & 4788 & 4030 & 13480 \\
\hline$[4,4]$ & 5810 & 5.2126 & 7492 & 5638 & 25150 \\
\hline$[5,5]$ & 9660 & 8.4177 & 10844 & 7678 & 40708 \\
\hline$[6,6]$ & 14482 & 12.3786 & 14844 & 10150 & 60154 \\
\hline$[7,7]$ & 20276 & 17.0954 & 19492 & 13054 & 83488 \\
\hline$[8,8]$ & 27042 & 22.5682 & 24788 & 16390 & 110710 \\
\hline$[9,9]$ & 34780 & 28.7969 & 30732 & 20158 & 141820 \\
\hline$[10,10]$ & 43490 & 35.7816 & 37324 & 24358 & 176818 \\
\hline
\end{tabular}

TABLE 5: Numerical results of indices for vanadium carbide.

\begin{tabular}{|c|c|c|c|c|c|}
\hline$[m, n]$ & $\operatorname{ABC}^{v e}(H)$ & $\mathrm{GA}^{v e}(H)$ & $\mathrm{H}^{v e}(H)$ & $\chi^{v e}(H)$ & $\mathrm{R}^{v e}(H)$ \\
\hline$[1,1]$ & 4.7074 & 10.8570 & 1.7088 & 1.8550 & 1.0319 \\
\hline$[2,2]$ & 13.2896 & 32.0346 & 3.2409 & 6.1227 & 2.7223 \\
\hline$[3,3]$ & 25.7590 & 65.2122 & 5.4396 & 12.3903 & 5.0793 \\
\hline$[4,4]$ & 42.1157 & 110.3898 & 8.3050 & 20.6579 & 8.1030 \\
\hline$[5,5]$ & 62.3597 & 167.5674 & 11.8370 & 30.9255 & 11.7933 \\
\hline$[6,6]$ & 86.4910 & 236.7450 & 16.0357 & 43.1932 & 16.1503 \\
\hline$[7,7]$ & 114.5096 & 317.9226 & 20.9011 & 57.4608 & 21.1740 \\
\hline$[8,8]$ & 146.4155 & 411.1002 & 26.4332 & 73.7284 & 26.8643 \\
\hline$[9,9]$ & 182.2087 & 516.2779 & 32.6319 & 91.9961 & 33.2213 \\
\hline$[10,10]$ & 221.8893 & 633.4555 & 39.4973 & 112.2637 & 40.2450 \\
\hline
\end{tabular}

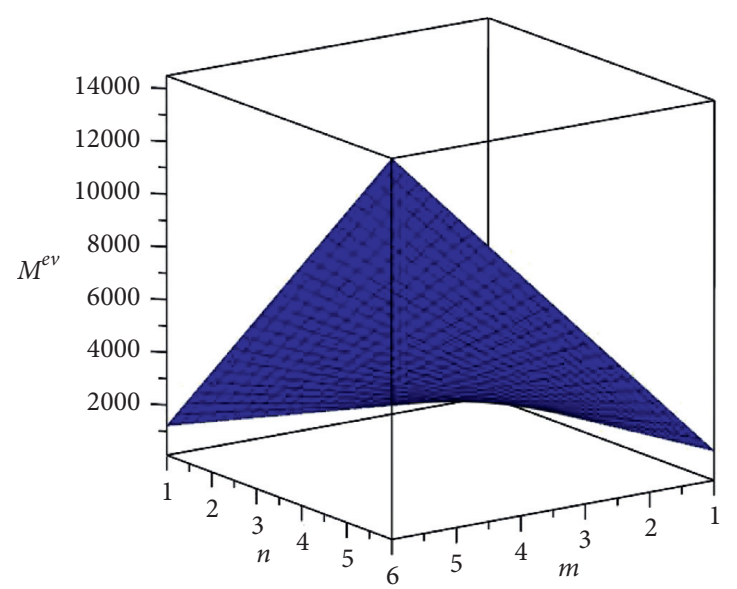

(a)

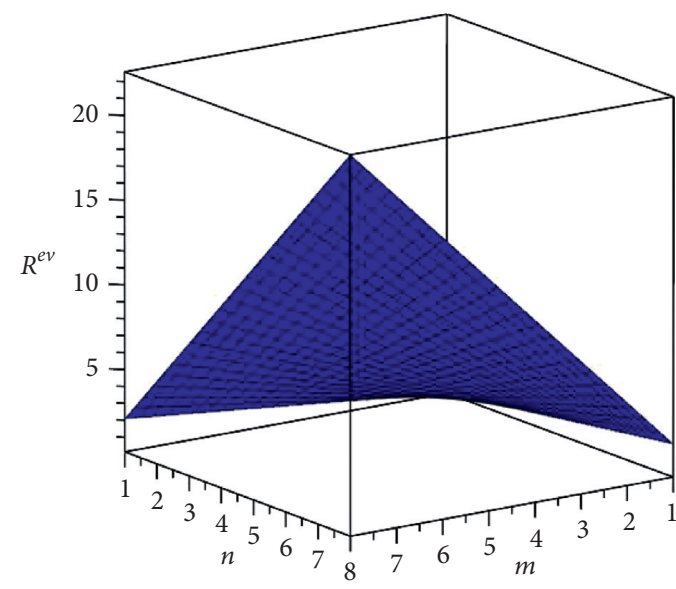

(b)

Figure 2: 3D plot of (a) $\mathrm{M}^{e v}(V C[m, n])$ (b) $\mathrm{R}^{e v}(V C[m, n])$.

\section{Numerical Results and Discussion}

Topological indices are used as vital tools for the analysis of chemicals, given the essential topology of chemical structures. Zagreb-type indices are used to calculate the total $\pi$-electronic energy of molecules [32]. The Randich index is commonly used to determine the chemical similarity of molecular compounds, as well as to calculate the boiling point and Kovaz constants of molecules [33]. The atom-bond connectivity index (ABC) provides a very good correlation for calculating strain energies as well as for the stability of linear and branched chemical structures [34]. It can be seen from Table 4 and 5 and Figures 2-6 of indices, an increase in the value of $m$ and $n$ raises the values of topological descriptors for vanadium carbide structure. 


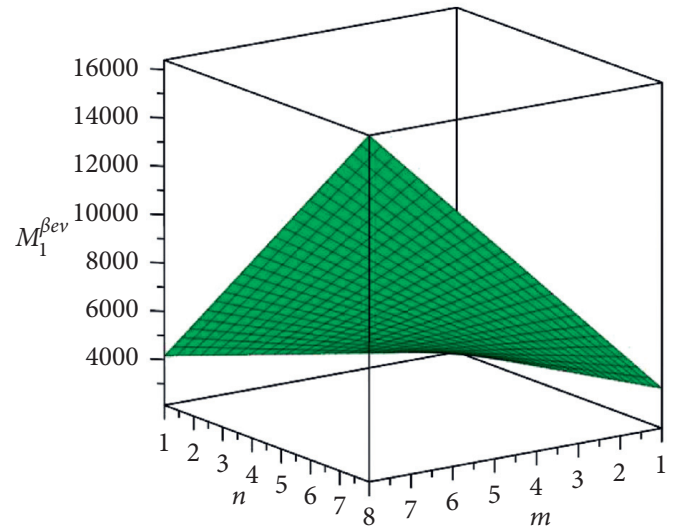

(a)

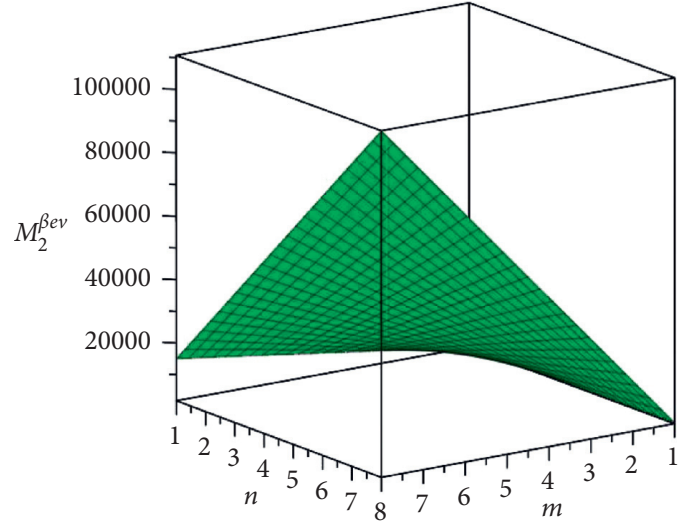

(b)

Figure 3: 3D plot of (a) $\mathbb{M}_{1}^{\beta v e}(V C[m, n])(b) \mathbb{M}_{2}^{\beta v e}(V C[m, n])$.

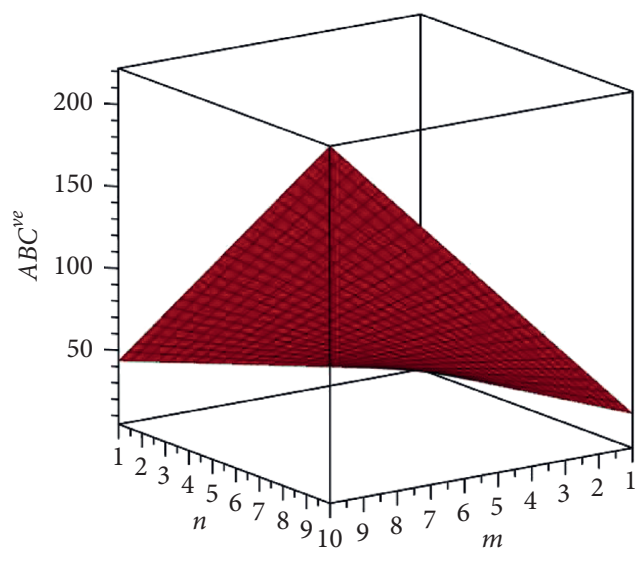

(a)

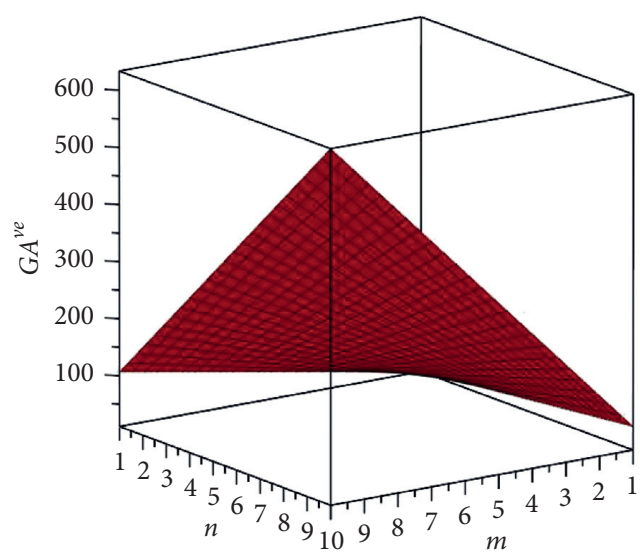

(b)

Figure 4: 3D plot of (a) $\mathrm{ABC}^{v e}(V C[m, n])(\mathrm{b}) \mathrm{GA}^{v e}(V C[m, n])$.

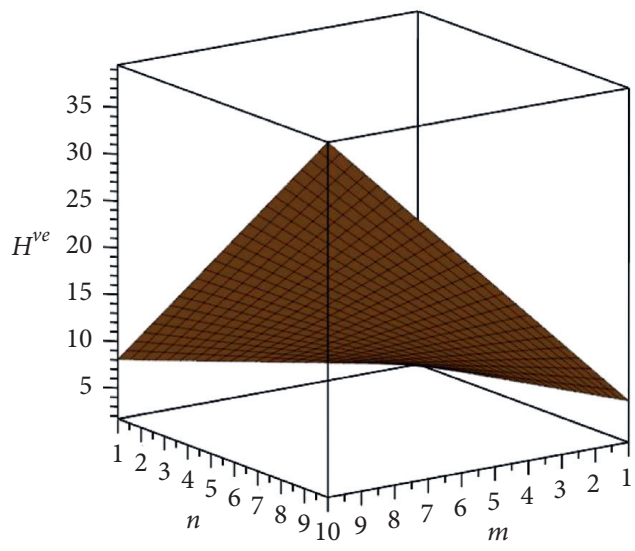

(a)

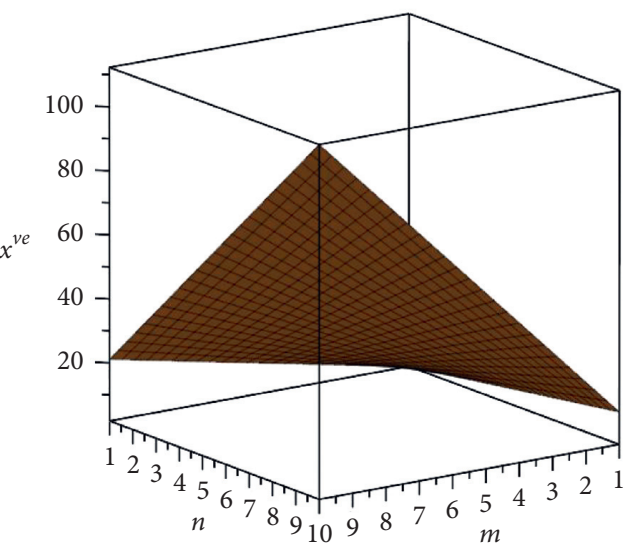

(b)

Figure 5: 3D plot of (a) $\mathrm{H}^{v e}(V C[m, n])$ (b) $\chi^{v e}(V C[m, n])$. 


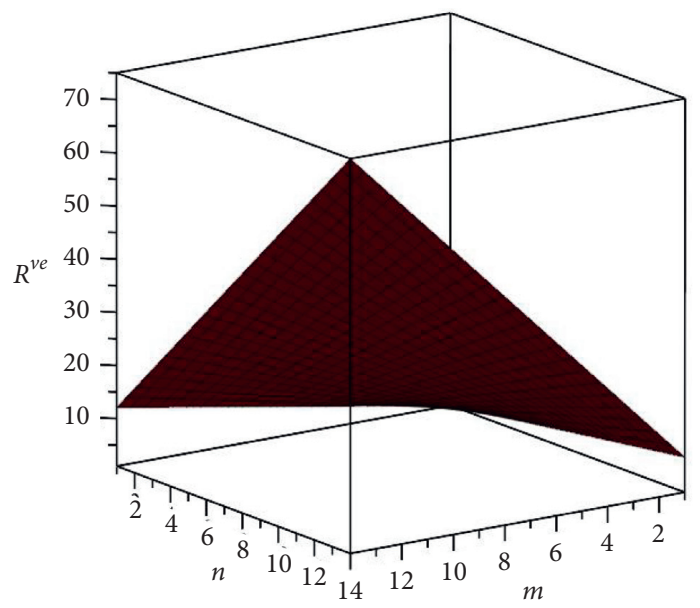

Figure 6: Graphical representation of $\mathrm{R}^{v e}(\operatorname{VC}[m, n])$.

\section{Conclusion}

Graphs invariants are calculated by some well-known topological indices which are important tools for resembling and forecasting the properties of chemical compounds in QSPRs and the QSARs. The TI is a numerical measure that represents the biological, physical, and chemical properties of molecules such as boiling, melting, and flickering point; moisture; and forming heat. In this paper, we have computed the ev degree and ve degree-based topological indices with graphical representations for the molecular structure of vanadium carbide for a better understanding of pharmaceutical, physical, chemical, and biological properties.

\section{Data Availability}

No data were used to support this study.

\section{Disclosure}

This research was carried out as a part of employment of the authors.

\section{Conflicts of Interest}

The authors hereby declare that there are no conflicts of interest regarding the publication of this paper.

\section{References}

[1] B. . Sahin and S. Ediz, "On ev-degree and ve-degree topological indices," Iranian Journal of Mathematical Chemistry, vol. 9, no. 4, pp. 263-277, 2018.

[2] S. Hosamani, D. Perigidad, S. Jamagoud, Y. Maled, and S. Gavade, "QSPR analysis of certain degree based topological indices," Journal of Statistics Applications \& Probability, vol. 6, no. 2, pp. 361-371, 2017.

[3] S. Ediz, "Predicting some physicochemical properties of octane isomers: a topological approach using ev-degree and vedegree Zagreb indices," 2017, https://ui.adsabs.harvard.edu/ link_gateway/2017arXiv170102859E/arXiv:1701.02859.
[4] H. Wiener, "Structural determination of paraffin boiling points," Journal of the American Chemical Society, vol. 69, no. 1, pp. 17-20, 1947.

[5] M. Randic, "Characterization of molecular branching," Journal of the American Chemical Society, vol. 97, no. 23, pp. 6609-6615, 1975.

[6] I. Gutman and N. Trinajstić, "Graph theory and molecular orbitals. Total $\varphi$-electron energy of alternant hydrocarbons," Chemical Physics Letters, vol. 17, no. 4, pp. 535-538, 1972.

[7] S. . Ediz, "Predicting some physicochemical properties of octane isomers: a topological approach using ev-degree and ve-degree Zagreb indices," 2017.

[8] Y.-X. Li, A. Rauf, M. Naeem, M. Ahsan Binyamin, and A. Aslam, "'Valency-based topological properties of linear hexagonal chain and hammer-like benzenoid," Complexity, p. 2021, 2021.

[9] S.-B. Chen, A. Rauf, M. Ishtiaq, M. Naeem, and A. Aslam, "On ve-degree- and ev-degree-based topological properties of crystallographic structure of cuprite $\mathrm{Cu} 2 \mathrm{O}$," Open Chemistry, vol. 19, no. 1, pp. 576-585, 2021.

[10] S. Shirakol, M. Kalyanshetti, M. Sunilkumar, and Hosaman, "QSPR analysis of certain distance based topological indices," Applied Mathematics and Nonlinear Sciences, vol. 4, no. 2, pp. 371-386, 2019.

[11] S. J. Patel, D. Ng, and M. S. Mannan, "QSPR flash point prediction of solvents using topological indices for application in computer aided molecular design," Industrial \& Engineering Chemistry Research, vol. 48, no. 15, pp. 7378-7387, 2009.

[12] J. C. Dearden, "The use of topological indices in QSAR and QSPR modeling," in Advances in QSAR Modeling, Challenges and Advances in Computational Chemistry and Physics, pp. 57-88, Springer, Cham, Switzerland, 2017.

[13] W. Xing, F. Meng, and R. Yu, "A new type of vanadium carbide V5C3 and its hardening by tuning Fermi energy," Scientific Reports, vol. 6, no. 1, pp. 21794-21799, 2016.

[14] A. VahidMohammadi, A. Hadjikhani, S. Shahbazmohamadi, and M. Beidaghi, "Two-dimensional vanadium carbide (MXene) as a high-capacity cathode material for rechargeable aluminum batteries," ACS Nano, vol. 11, no. 11, pp. 11135-11144, 2017.

[15] Y. Guan, S. Jiang, Y. Cong et al., "A hydrofluoric acid-free synthesis of $2 \mathrm{D}$ vanadium carbide (V2C) MXene for 
supercapacitor electrodes," 2D Materials, vol. 7, no. 2, Article ID 025010, 2020.

[16] D. Huang, Y. Xie, D. Lu et al., "Demonstration of a white laser with V2C MXene-based quantum dots," Advanced Materials, vol. 31, pp. 1901117-24, 2019.

[17] D. B. West, Introduction to Graph Theory, Vol. 2, PrenticeHall, Upper Saddle River, NJ, USA, 2001.

[18] M. Chellali, T. W. Haynes, S. T. Hedetniemi, and T. M. Lewis, "On ve-degrees and ev-degrees in graphs," Discrete Mathematics, vol. 340, no. 2, pp. 31-38, 2017.

[19] A. N. A. Koam, A. Ahmad, and A. A. Ahamdini, "Computation of vertex-edge degree based topological descriptors for hex-derived networks," IEEE Access, 2021.

[20] E. A. Refaee and A. Ahmad, "A study of hexagon star network with vertex-edge-based topological descriptors," Complexity, vol. 2021, 2021.

[21] A. Ahmad, "Comparative study of ve-degree and ev-degree topological descriptors for benzene ring embedded in P-TypeSurface in 2D network," Polycyclic Aromatic Compounds, pp. 1-10, 2020.

[22] J. Zhang, M. K. Siddiqui, A. Rauf, and M. Ishtiaq, "On vedegree and ev-degree based topological properties of single walled titanium dioxide nanotube," Journal of Cluster Science, pp. 1-12, 2020.

[23] B. Horoldagva, K. Ch Das, and T.-A. Selenge, "On ve-Degree and ev-Degree of Graphs," Discrete Optimization, vol. 31, pp. 1-7, 2019.

[24] Te-H. Fang, S.-R. Jian, and D.-S. Chuu, "Nanomechanical properties of $\mathrm{TiC}, \mathrm{TiN}$ and $\mathrm{TiCN}$ thin films using scanning probe microscopy and nanoindentation," Applied Surface Science, vol. 228, no. 1-4, pp. 365-372, 2004.

[25] H. G. Prengel, W. R. Pfouts, and A. T. Santhanam, "State of the art in hard coatings for carbide cutting tools," Surface and Coatings Technology, vol. 102, no. 3, pp. 183-190, 1998.

[26] K. H. Lo, F. T. Cheng, C. T. Kwok, and H. Chung Man, "Mprovement of cavitation erosion resistance of AISI 316 stainless steel by laser surface alloying using fine WC powder," Surface and Coatings Technology, vol. 165, no. 3, pp. 258-267, 2003.

[27] B. Chicco, W. E. Borbidge, and E. Summerville, "Experimental study of vanadium carbide and carbonitride coatings," Materials Science and Engineering: A, vol. 266, no. 1-2, pp. 62-72, 1999.

[28] S. A. Hassanzadeh-Tabrizi, "Homa Hosseini Badr, and Sara Alizadeh. "In situ synthesis of vanadium carbide-copper nanocomposite by a modified mechanochemical combustion method," Ceramics International, vol. 42, no. 8, pp. 9371-9374, 2016.

[29] D. Ferro, J. V. Rau, A. Generosi, V. Rossi Albertini, A. Latini, and S. M. Barinov, "Electron beam deposited VC and NbC thin films on titanium: hardness and energy-dispersive X-ray diffraction study," Surface and Coatings Technology, vol. 202, no. 10, pp. 2162-2168, 2008.

[30] L. B. Wang, Z. F. Chen, Y. Zhang, and W. P. Wu, International Journal of Refractory Metals and Hard Materials, p. 2004, 2009.

[31] A. Aouni, P. Weisbecker, T. Huu Loi, and E. Bauer-Grosse, "Search for new materials in sputtered V1-xCx films," Thin Solid Films, vol. 469, pp. 315-321, 2004.

[32] I. Gutman, B. Ruščić, N. Trinajstić, and C. F. Wilcox Jr., "Graph theory and molecular orbitals. XII. Acyclic polyenes," The Journal of Chemical Physics, vol. 62, no. 9, pp. 3399-3405, 1975.
[33] N. Nikolova and J. Jaworska, "Approaches to measure chemical similarity-a review," QSAR \& Combinatorial Science, vol. 22, no. 9-10, pp. 1006-1026, 2003.

[34] E. Estrada, L. Torres, L. Rodriguez, and I. Gutman, “An atombond connectivity index: modelling the enthalpy of formation of alkanes," 1998. 